\title{
LA RELEVANCIA DE LA SEMIÓTICA DE CH. S. PEIRCE EN LA CONSTITUCIÓN DE UNA PRAGMÁTICA DE LA LITERATURA
}

\author{
Francisco Vicente Gómez
}

(Universidad de Murcia)

Referirse hoy a las relaciones entre semiótica y literatura, y más en concreto a la aportación de Ch. S. Peirce al tema en cuestión requiere al menos algunas precisiones iniciales, pues la presencia y aun la pujanza de la semiótica en los estudios literarios no es reciente, ni tampoco la de Ch. S. Peirce, si bien en este último caso es necesario anotar ya que la versión castellana más amplia de sus escritos lógico-semióticos data sólo de 1987.

En primer lugar, la inclusión de los textos literarios bajo el dominio de la semiótica ha permitido observar y caracterizar la literatura como hecho comunicativo. Así lo ponía de manifiesto F. Lázaro Carreter en un trabajo de 1976: 
Una caracterización general y moderadamente aceptable del hecho literario sólo ha sido posible desde el momento en que se ha reconocido en las obras artísticas el carácter de signos, y se han inscrito por tanto en el campo de acción de la semiótica. Ello implica que se consideren actos de comunicación ... (Lázaro Carreter 1976: 176).

En segundo lugar, la presencia de la figura de Ch. S. Peirce debe ser considerada no 'ex novo' sino desde la óptica de la relectura de sus principales escritos semióticos; relectura que puede enriquecer considerablemente cuanto la semiótica había ya desarrollado incorporando perspectivas diversas y abriendo caminos nuevos. Se trata, pues, de una 'relectura' que acompaña el 'redescubrimiento' que de su figura y su obra - con nuevas traducciones y recopilaciones de sus trabajos- ha tenido lugar en la década de los años 80, al amparo de una generalizada necesidad científica de hallar el fundamento epistemológico de las ciencias humanas en general, y de los estudios literarios en particular (Bonfantini 1982: 45).

El encuentro entre los principales retos que tienen hoy planteados los estudios literarios y los caminos que la aportación de $\mathrm{Ch}$. S. Peirce abre a la misma perspectiva semiótica, nos puede proporcionar el adecuado punto de partida al tema que nos ocupa: la relevancia de la semiótica de Ch. S. Peirce en la constitución de una pragmática de la literatura.

El primer reto que a la semiótica plantearon los textos literarios fue pronto afrontado con resultados bastante satisfactorios. Libros como los de María Corti (1976) y María del Carmen Bobes Naves (1975) en España son suficiente testimonio. Los textos literarios propician una situación comunicativa ciertamente particular; ésta se caracteriza por no existir una co-presencia física de autor y lector, dando lugar ello a un tipo de comunicación diferida que va del autor al texto y del texto al lector. En esta comunicación autor y lector además de no poseer el mismo sistema y código culturales, están con toda probabilidad muy alejados tanto en el tiempo como en el espacio (Segre 1985: 42-43).

La peculiar situación comunicativa de los textos literarios origina una definición de los textos literarios como textos menos dependientes de aquellos factores que apuntan todos a la inmediatez de la situación (la deixis espacio-temporal, la personal, la modalidad, etc.), y mucho más dependientes de aquellos otros elementos que hacen posible tanto la es- 
critura como la lectura de un texto a pesar de la no co-presencia de autor y lector (los saberes culturales disponibles en forma de códigos que en mayor o menor medida comparten).

Los signos literarios, pues, están más necesitados de una codificación múltiple (Corti 1976: 121-148), la cual mediante la dinamización textual de todos sus componentes (García Berrio 1978: 148), o lo que es lo mismo la polifuncionalidad de todos tematiza la intersubjetividad de estos signos hasta el punto de hacerlos garantes de sí mismos (Pozuelo Yvancos 1988: 76-83).

\section{III}

Descrita y caracterizada en estos términos la comunicación literaria, así como cada uno de sus componentes (Pagnini 1980), quedaban sin embargo sin resolver la delimitación de la situación verbal en la que tiene lugar y la concreción de la dinámica interactiva en la que entran todos sus elementos, a saber, autor, lector, texto y contexto.

La semiótica de Ch. S. Peirce, frente a la semiótica de corte descriptivo auspiciada por F. de Saussure, definida como una 'semiótica de los códigos' y frente a la semiótica de tinte psicoanalítico elaborada por J. Kristeva, calificada como una 'semiótica de significación', es esencialmente cognoscitiva e interpretativa, y posibilita un tratamiento adecuado de los dos aspectos que quedaban pendientes de concreción en la caracterización comunicativa de la literatura (Bonfantini 1982: 28-45).

La semiótica de Ch. S. Peirce es esencialmente cognoscitiva porque a la «concezione della conoscenza come perfetta specularità Peirce oppone la concezione della conoscenza quale sempre, necessariamente, intrinsecamente, interpretativa e ipotetica» (Bonfantini 1980: XXII). Y es también esencialmente interpretativa porque en Peirce «la generazione del senso non viene più intesa propriamente come produzione di senso,..., bensi costituzione e inferenza di senso, interpretazione» (Bonfantini 1982: 45).

La definición comunicativa de los signos viene indicada por su situación de 'uso'; «el significado de una palabra es su uso en el lenguaje», dice Ludwig Wittgenstein (1953: \#43), este uso tiene lugar en el transcurso de una actividad verbal concreta y única, que tiene sus propias reglas (Wittgenstein 1953: \#23). La especificación del significado verbal en el 'uso' la explicaba Ch. S. Peirce como una relación de 'intersubjetividad' a partir de la noción de 'interpretante': 
Un signo o representamen es algo que representa algo para alguien en algún aspecto o carácter. Se dirige a alguien, es decir, crea en la mente de esa persona un signo equivalente o quizás aún, más desarrollado. A este signo creado, yo lo llamo el Interpretante del primer signo. El signo está en lugar de algo, su objeto. Representa este objeto no en todos sus aspectos, sino con referencia a una idea que he llamado a veces el fundamento del representamen. (Peirce 2.228).

La intersubjetividad verbal de la que surge el significado de los textos - literarios también- es en la semiótica peirceana un rasgo inherente a la propia definición de 'semiosis', objeto de la Semiótica en tanto que «doctrina cuasi-necesaria, o formal, de los signos» (Peirce 2.227). En los siguientes términos define Ch. S. Peirce la 'semiosis':

Con 'semiosi', invece, intendo un' azione o influenza che è, o implica, una cooperazione di tre soggetti, il segno, il suo oggetto e il suo interpretante, tale che questa influenza trirelativa non si possa in nessun modo risolvere in azioni fra coppie. (Peirce, 5.484).

Es preciso advertir que «this extension of the conception of Semiosis [cognitivo-interpretativa] — como explica Dan Nesher- comes... as a natural conclusion of the reconstruction of Peirce's pragmaticist philosophy» (Nesher 1990: 1). A su vez, como señalase Antonio Tordera, la conexión «entre el pragmatismo y la semiótica, que se realiza a través de la teoría de los interpretantes y del hábito, conlleva varias consecuencias. La principal es la posibilidad de elaborar una semiótica pragmática, no como una mera sistematización de todos los signos posibles, sino como algo que aporta elementos de solución al problema de la producción de significados» (Tordera 1978: 44).

La explicación del significado a través de la noción de 'interpretante' (Eco 1979: 48;57), noción hipotética que implica procesos de inferencia (Peirce 5.274), supone afirmar que nuestro conocimiento posee un carácter aproximado (Herrero 1987: 15). De este modo lo argumenta el propio Ch. S. Peirce:

... toda proposición que estemos en condiciones de hacer acerca del mundo real debe ser de carácter aproximado: no podemos tener nunca el derecho de afirmar que cualquier verdad es exacta. $\mathrm{La}$ aproximación debe ser la estructura con la cual corresponde construir nuestra filosofía (Pęirce 1.404).

El carácter aproximado que posee el 'interpretante' descansa en una adecuación parcial, abductiva del signo respecto del objeto, y es fruto de 
la mediación creadora del hombre (Th. A. Sebeok y J. U. Sebeok 1989: 37-40). Ch. S. Peirce es categórico al respecto en sus afirmaciones:

\begin{abstract}
Non esiste [la cosa-in-sé]. Cioè non vi è cosa che sia in se stessa nel senso di non essere relativa alla mente, sebbene le cose che sono relative alla mente senza dubbio esistono anche al di là di questa relazione. Le cognizione che cosí arrivano a noi attraverso questa serie infinita di induzioni e ipotesi. (Peirce 5.311).
\end{abstract}

Además, dado que «tuttavia no abbiamo esperienza di semiosi in cui no si abbia modificazione della coscienza,... l'interpretante sia por lo meno un analogo piuttosto stretto di una modificazione di coscienza» (Peirce, 5.485). Es por ello que toda ocurrencia comunicativa es constitutiva de significado y que la semiosis se resuelve en un proceso que no tiene fin, y en la que la condición de Tercero del interpretante hace que ésta se resuelva en una 'semiosis ilimitada':

El significado de una representación puede ser tan sólo una representación. De hecho, no es más que la propia representación, pensada como si estuviera despojada de un ropaje sin importancia. Pero no se puede eliminar nunca por completo este ropaje; sólo se lo cambia por algo más diáfano. Por último, el interpretante es tan sólo otra representación a la cual se entrega la antorcha de la verdad, y en calidad de representación tiene a su vez su interpretante. He aquí otra serie infinita (Peirce 1.339).

Para Ch. S. Peirce todo signo es 'interpretante' de otro signo. Pero la comunicación es socialmente intersubjetiva, y la 'semiosis' un 'interpretante final': aquel que posibilita que un hablante y un oyente, desde la especificidad histórica de sus respectivos roles socio-culturales, se entiendan:

... un comportamiento del medesimo genere, piú volte reiterato nel quadro di combinazioni simili di preceti e di fantasie, produce l'abito, cioè la tendenza a comportarsi effetivamente in un modo simile in circostanze simili nel futuro... le reiterazioni nel mondo interno - reiterazioni fantasticate-, se ben intensificate da uno sforzo diretto producono abiti, ...; e questi abiti avranno il potere di influenzare el comportamento effettivo nel mondo esterno... (Peirce 5.487).

El límite que impone el 'interpretante final' como regularidad, como hábito de comportamiento verbal y que posibilita la intersubjetividad verbal, permite referir usos verbales a situaciones comunicativas típicas, es decir, permite correlatar regularmente las variedades de textos lingüísticos a categorías específicas y recurrentes de rasgos sociosituacionales. 
La recurrencia histórica de estas clases textuales las convierte en interpretantes finales o entornos interpretativos del texto que se inscribe en una de ellas, pues éstas insertan y ordenan el texto individual dentro del devenir histórico del saber textual de una cultura con el fin de hacerlo comprensible.

La pragmática, como disciplina semiótica que básicamente indaga las relaciones entre los signos y los hablantes (Morris 1971: 67), entre los textos y los contextos (van Dijk 1977: 272; cf. Levinson 1983: 18-23), encuentra en el estudio de la interacción texto-entorno textual una de las vías de especificación de la intersubjetividad útil para la interpretación de los textos literarios.

La pragmática literaria, sin menoscabo de ulteriores especificaciones, centra su atención en las relaciones implícitas que autor y lector mantienen, relaciones implícitas que son el imprescindible apoyo de aquellas otras relaciones - las inmediatas-que sí explicita el texto. Sobre estas relaciones implícitas tiene gran capacidad de decisión el entorno interpretativo constituido por el saber textual que envuelve al autor y al lector; constituyen su auténtica realidad.

La intersubjetividad que especifica el texto, su coherencia (Conte 1988: 29) —incluidos el autor y el lector modelo (Eco 1979: 87-90)-, es una proyección histórica, inferencial, nunca directa, del entendimiento cultural que pueden llegar a tener autor y lector. Ese entendimiento cultural es el que configura el contexto.

La cooperación textual (texto-contexto) que investiga una pragmática así concebida encuentra en la perspectiva semiótica peirceana el soporte adecuado para su definición, pues Ch. S. Peirce privilegia la 'semiosis', entendida como constitución de sentido, en lugar de la semiótica sistemática y descriptiva, la 'diacronía' en lugar de la 'sincronía', al 'interpretante' en lugar del 'código' fijo e idéntico a sí mismo, la 'interpretación' en lugar de la 'representación' (Bonfantini 1982: 41).

Desde una perspectiva pragmática operativa y no ontológica, nociones como las de 'código', en el sentido de 'código institucional' o 'hipercódigo' (Eco 1979: 316), 'frame testual' o 'text type' (Schlothaus 1988: 76), pueden ser de una gran utilidad para explicar el funcionamiento interpretativo (Eco 1979: 109-122) de los entornos enciclopédicos o contextos constituidos por clases de textos precedentes de costumbres o há- 
bitos textuales, considerados como interpretantes finales.

El camino semiótico elegido permite recuperar la historicidad y el contexto socio-cultural que son connaturales a todo proceso semiósico. Este proceso semiósico, concebido como cualquier actividad humana que crea sentido, esto es, como toda actividad dinámica realizada por un sujeto que pone en relación una forma y un sentido, sólo puede tener lugar dentro de una situación pragmática de uso (Bobes Naves 1989: 118-121).

Sin necesidad de desembocar en el extremo deconstruccionista de algunas explicaciones de la 'semiosis ilimitada' de Ch. S. Peirce (Ponzio 1984: 18-22), la recuperación que ésta hace del significado contextual y la importancia que concede a éste en la constitución del significado a través de la noción de 'interpretante final', posibilita hacer un uso teórico y analítico muy razonable de la noción de 'contexto' en la consideración de la semiosis literaria (Eco 1990: 267), pues éste no es considerado como un elemento exterior sino como un factor constitutivo del propio signo.

La semiótica de Ch. S. Peirce precisamente por su acentuado carácter cognitivo e interpretativo aporta una concepción radicalmente nueva respecto de cómo entendían la relación entre signo y objeto tanto la tradición racionalista como empirista: la perspectiva semiótica de $\mathrm{Ch}$. S. Peirce es intrínsecamente interpretativa e hipotética (Pérez Carreño 1988: 30-32); el camino de establecimiento de las relaciones entre signo y objeto es antes inferencial - contextual- que intuitivo, y se resuelve en un flujo de aproximaciones en las que «ogni pensiero precedente suggerisce qualcosa al pensiero seguente» (Peirce 5.284).

Explicando a Ch. S. Peirce, dice U. Eco que «interpretare un segno significa prevedere -idealmente - tutti i contesti possibili in cui può essere inserito» (Eco 1990: 267). A esta conclusión ha llegado U. Eco tras describir la actuación del 'interpretante' del modo que sigue:

Ogni espressione deve venire interpretata da un'altra espressione, e cosi via, all'infinito;

(..)

nel corso di questo processo semiosico, il significato socialmente riconosciuto delle espressioni cresce attraverso le interpretazioni a cui esse vengono sottoposte in diversi contesti e in diverse circostanze storiche; il significato completo di un segno no può che essere la registrazione storica del lavoro pragmatico che ha accompagnato ogni apparizione contestuale. (Eco 1990: 267).

La noción de 'interpretante' de Ch. S. Peirce no proporciona una definición precisa de interpretación, pero sí elabora una perspectiva capaz de 
dar un fundamento general a otras definiciones más elaboradas como, por ejemplo, la que da S. Schmidt. Para este autor la interpretación construye relaciones «between the conceptual structures called forth by the text and the conceptual network that constitutes one's own experiental world... or relating conceptual structures called forth by the next and 'frames of reference'» (Schmidt 1982: 256). La cooperación texto-contexto, constructora de la coherencia global de un texto, no es determinista ni está unívocamente señalada, antes bien es interpretativa.

La lógica interpretativa que ayuda a comprender el funcionamiento de los distintos contextos que contribuyen a elaborar el entorno textual sobre el que se apoyan los textos (el socio-histórico, el cultural y el literario, principalmente) hace posible que éstos reformulen de una manera totalmente nueva los llamados 'accesos extrínsecos' del estudio de la literatura (biografismo, historias de la literatura, sociologías de la literatura, pragmáticas, etc.), quedando articulados dentro de un modelo -el semiótico-textual-, de una perspectiva - la interpretativa-, así como clarificando el camino hacia su relevancia textual: ser soportes de coherencia.

Muy particularmente la 'lógica interpretativa' definida por la semiótica peirceana abre un camino esperanzador en la constitución de una pragmática literaria a partir de elementos culturales tales como las convenciones estilísticas y genéricas, pues la noción de interpretante, como dice U. Eco, «muestra de qué manera los procesos semióticos... circunscriben asintóticamente los significados, sin llegar nunca a 'tocarlos' directamente, pero volviéndolos de hecho accesibles mediante otras unidades culturales» (Eco 1984: 131), por medio de procesos como las hipercodificaciones.

Desde la optica semiótica peirceana se vislumbra mejor la fecundidad que el modelo tipológico de descripción de la cultura instaurado por la semiótica soviética puede ofrecer para la delimitación del contexto literario y la definición de la pragmática literaria a partir de las unidades culturales mencionadas. Su amplia capacidad conceptualizadora absorbe de un modo típico, relevante la diversa realidad sociosituacional dispersa en los diferentes contextos. Así lo pone de manifiesto C. Segre en el texto siguiente:

la influencia, y a menudo incluso el impacto, de las fuerzas historicas (principalmente económicas) actúa mucho más determinantemente sobre el conjunto del sistema cultural que sobre cada uno de los textos en particular. Por tanto, el estudio de la cultura es el que puede mediar entre el estudio histórico y el de los textos (Segre 1985: 145). 


\section{Referencias bibliográficas}

Bobes Naves, M. C. (1975): Gramática de «Cántico». Madrid: Cupsa.

- (1989): La semiología. Madrid: Síntesis.

Bonfantini, M. A. (1980): «Introduzione: la semiotica cognitiva di Peirce». En Semiotica, Ch. S. Peirce, XXI-LII. Testi scelti e introdotti da M. A. Bonfantini, L. Grassi e R. Grazia. Torino: Einaudi, 1980.

- (1982): «Le tre tendenze semiotiche del novecento». En Semiotica ai media, 27-57. Bari: Adriatica Editrice, 1984.

CORTI, M. (1976): Principi della comunicazione letteraria. Introduzione alla semiotica della letteratura. Milano, Bompiani.

DiJK, T. A. VAN (1977): Texto y contexto. Semántica y pragmática del discurso. Madrid: Cátedra, 1984.

Eco, U. (1979): Lector in fabula. La cooperación interpretativa en el texto narrativo. Barcelona: Lumen, 1981.

- (1984): Semiótica y filosofía del lenguaje. Barcelona: Lumen, 1990.

- (1990): I limiti dell'interpretazione. Milano: Bompiani.

García Berrio, A. (1979): «Lingüística, literaridad/poeticidad (Gramática, Pragmática, Texto)». 1616. Anuario de la Sociedad Española de Literatura General y Comparada, II, 125-170.

Herrero, A. (1988): Semiótica y creatividad. La lógica abductiva. Madrid: Palas Atenea.

LÁZARo CARRETER, F. (1976): «La literatura como fenómeno comunicativo». En Estudios de lingüística, 173-192. Barcelona: Crítica, 1981.

Levinson, S. (1983): Pragmática. Barcelona: Teide, 1989.

MorRIs, CH. S. (1971): Fundamentos de la teoría de los signos. Barcelona: Paidós, 1985.

NESHER, D. (1990): «Understanding sign semiosis as cognition and as self-conscious process: A reconstruction of some basic conceptions in Peirce's semiotics». Semiotica, 79-1/2, 1-49.

PaGnini, M. (1980): Pragmatica della letteratura. Palermo: Selleiro Editore, 1988.

Peirce, Ch. S. (1868-1935): Semiotica. Testi scelti e introdotti da M. A. Bonfantini, L. Grassi e R. Grazia. Torino: Einaudi, 1980.

- (1868-1935): Obra lógico-semiótica. Edición de Armando Sercovich. Madrid: Taurus, 1987.

PÉrez CARREÑo, F. (1988). Los placeres del parecido. Icono y representación. Madrid: Visor.

PONZIO, A. (1984): «La semiotica tra Peirce e Bachtin». En Interpretazione e scrittura. Scienza dei segni ed eccedenza letteraria, 15-52. Verona: Bertani, 1986.

Pozuelo Yvancos, J. M. (1988): Teoría del lenguaje literario. Madrid: Cátedra.

Schlothaus, W. (1989): «Conditioning Factors of Textual Understanding». En Comprehension of Literary Discourse, D. Meutsch and R.

SEBEOK, Th. A. y UMIKER-SEBEOK, J. (1983): «'Ya conoce usted mi método': una confrontación entre Charles S. Peirce y Sherlock Holmes». En El signo de los tres. Dupin, Holmes, Peirce, U. Eco y Th. A. Sebeok (eds.), 31-81. Barcelona: Lumen, 1989. Viehoft (eds.), 74-88. Berlin: Walter de Gruyter.

Segre, C. (1985): Principios de análisis del texto literario. Barcelona: Crítica.

Tordera, A. (1978): Hacia una semiótica pragmática. El signo en Ch. S. Peirce. Valencia: Fernando Torres Editor.

WITTGENSTEIN, L. (1953): Investigaciones filosóficas. México/Barcelona: UNAM/Crítica, 1988. 